\title{
Electron Holography, a Routine Application Workflow
}

\author{
E. Voelk1 ${ }^{1}$, Arturo Ponce ${ }^{2}$ \\ ${ }^{1 .}$ HoloWerk LLC, Germantown, USA. \\ 2. Department of Physics and Astronomy, University of Texas at San Antonio, San Antonio, USA.
}

1954 is the year the first electron hologram was recorded [1]. Its method remains and is a powerful cornerstone for imaging the phase of the electron wave. The modulation of the electron wave in its phase by the sample has recently created strong interest for, e.g., the live science community. The Zernike phase contrast, a predecessor of electron holography is spreading rapidly for its use with objects that typically generate little contrast in the electron microscope - although it's images are qualitative rather than quantitative. Differential phase contrast (DPC) has shown effective in obtaining the phase of the electron wave, especially for electric and magnetic fields. While DPC is quantitative, the technique needs further development in areas where quantum mechanical effects dominate contrast generation.

Results from recently available single-electron cameras have proven the superiority of imaging methods recording the phase of the electron wave: given the identical number of electrons incident on the sample, the phase can show detailed information where conventional intensity images yield nothing but noise [2]. Furthermore, phase imaging methods continue to gain attractiveness even in high-speed imaging where phase images continue to yield decent contrast even at the shortest exposure times.

Electron holography does come with its own limitations. Many of them can be dealt with by experience and/or skilful use of available technology. Key developments like improved transmission electron microscope (TEM) stability and accelerating computational speed, advances in parallel processing and the development of application focused software have a high impact on the usability of electron holography. These developments have allowed a phase resolution increase by more than an order of magnitude [3], while simultaneously simplifying the holography technique.

There are two distinct points in time: when research becomes enabled [3] and later when the enabler turns routine application [4]. We report that electron holography with high phase resolution today can be considered a routine activity. This is partially due to recognition and elimination of prejudices (e.g., for the required sampling rate for interference fringes). But, the real "game changers" are high speed parallel computing, fast cameras, stable microscopes and application software using such improvements.

Live phase imaging today runs at $>5$ frames /s. However, more than just speed is required to obtained high-quality data with ease. Application software provides feedback and guides helping the user achieve required data easily. Such guides need to quantify the stability of interference fringes, their drift and contrast and provide a quantity that characterizes the quality of the recorded holograms - related to the noise level in the phase image (thus defining the phase resolution in fractions of $2 \pi$ ). Figure 1 shows how such parameters are traced via live line plot, tracking fringe drift, fringe contrast and the Q- factor.

Allowing the application space to become "live" however is even more important. Examples of processes that can be attached to live phase imaging as separate processes (parallel computing) include: live biprism-Fresnel removal, live compensation of the drift of the interference fringes, live phase unwrap, live scaling of the phase image (e.g., rad to $\mathrm{nm}$ ), live display of magnetic induction and contour 
lines (see Figure 1), and any other feature like additionally attached line plots.

In terms of ease of use, it appears that the long-standing practice of astigmatic illumination for electron holography is going away. Easy acquisition of data stacks and fast averaging enable data acquisition with round illumination to yield phase resolutions sufficient for many applications.

As summary: the number of step for acquiring and processing holograms is significant and manageable by today's top of the line workstation. Here, the creation and optimization of a reliable workflow is key to turn a complex technique like holography into a routine and easy to use application. We believe electron holography just made that leap [4].

\section{References:}

[1] G. Möllenstedt, in "Introduction to Electron Holography", ed. E Voelkl, L F Allard and D C Joy, (Kluwer Academic, New York), p.1.

[2] E Voelkl, R Herring, B Bammes and D Hoyle, Microsc. Microanal. 21 (Suppl 3), 2015.

[3] E. Voelkl and Dong Tang, Ultramicroscopy 110 (2010), p. 447.

[4] The author acknowledges funding from HoloWerk LLC at www.HoloWerk.com

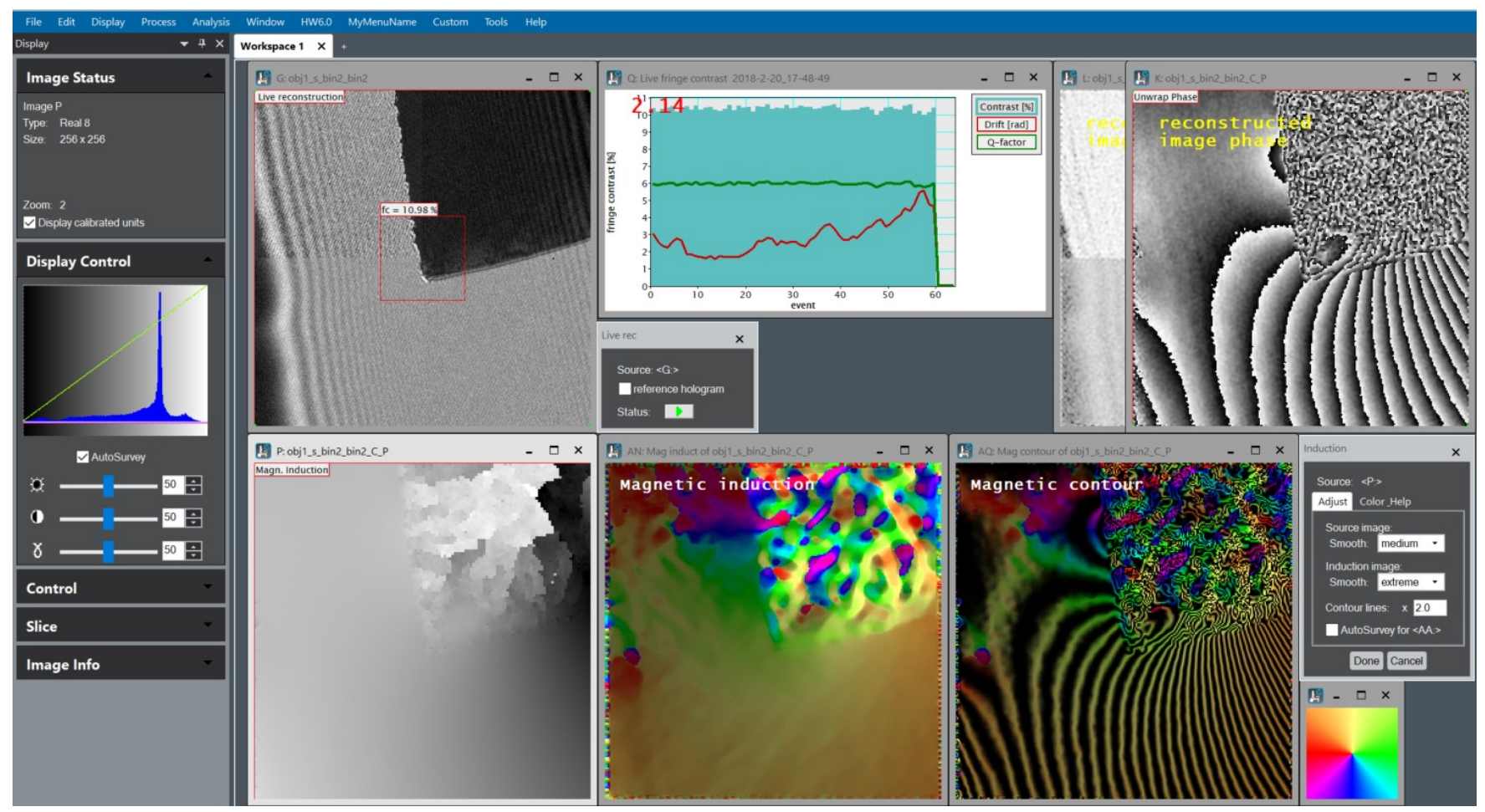

Figure 1: Live holography in action - overview of a multi-process task (left to right): acquisition and display of holograms at several frames/second; line plot tracing key parameters from live holograms; reconstruction proving live phase images; process unwrapping phase image; process computing and displaying magnetic induction profile and magnetic contour lines; control window and colour wheel. 